\title{
Preconception prediction of expectant fathers' mental health: 20-year cohort study from adolescence
}

Elizabeth Spry, Rebecca Giallo, Margarita Moreno-Betancur, Jacqui Macdonald, Denise Becker, Rohan Borschmann, Stephanie Brown, George C. Patton* and Craig A. Olsson*

We examined prospective associations between men's common mental disorders in the decades prior to offspring conception and subsequent paternal antenatal mental health problems. Data came from a prospective intergenerational cohort study which assessed common mental disorder nine times from age 14 to 29 years, and in the third trimester of subsequent pregnancies to age 35 years $(N=295$ pregnancies to 214 men). Men with histories of adolescent and young adult common mental disorders were over four times more likely to experience antenatal mental health problems. Future research identifying modifiable perinatal factors that counteract preconception risk would provide further targets for intervention.

\section{Declaration of interest}

None.

\section{Copyright and usage}

(c) The Royal College of Psychiatrists 2018. This is an Open Access article, distributed under the terms of the Creative Commons Attribution-NonCommercial-NoDerivatives licence (http://creativecommons.org/licenses/by-nc-nd/4.0/), which permits noncommercial re-use, distribution, and reproduction in any medium, provided the original work is unaltered and is properly cited. The written permission of Cambridge University Press must be obtained for commercial re-use or in order to create a derivative work.
Mental health problems affect one in ten men during their partner's pregnancy. ${ }^{1}$ These problems tend to persist into the postpartum period $^{2,3}$ and predict increasing severity of maternal depression symptoms from pregnancy to postpartum; ${ }^{2}$ in turn, paternal postpartum depression has been associated with adverse child mental health outcomes both via and independently of maternal depression. ${ }^{3,4}$ Early identification of men at risk of antenatal mental health problems could inform the development of targeted intervention strategies within existing preconception and perinatal healthcare systems. ${ }^{5,6}$ However, little is known about paternal risk factors, as prospective studies including data prior to pregnancy are scarce. Using data from a 20 -year, two-generation study, we aimed to examine the extent to which common mental disorders in the decades prior to conception predicted antenatal paternal mental health problems.

\section{Method}

\section{Participants}

The Victorian Intergenerational Health Cohort Study (VIHCS) is a prospective study of preconception predictors of child health. It arose from a cohort study commencing in 1992 in Victoria, Australia (the Victorian Adolescent Health Cohort Study; VAHCS). A close-to-representative sample of 1943 mid-secondary school students (943 male) was selected using a two-stage sampling procedure. Participants were assessed 6-monthly during adolescence (waves 1-6: mean age 14.9-17.4 years) and three times in young adulthood (waves 7-9: 20.7, 24.1 and 29.1 years).

Between 2006 and 2013, when participants were aged 29-35 years (encompassing median paternal age for Australian births ${ }^{8}$ ), the 1645 study members (773 male) still active in VAHCS were screened 6-monthly for participation in VIHCS. Study members were invited to complete telephone interviews in trimester three

* Joint senior authors
(VIHCS wave 1), 2 months' postpartum (VIHCS wave 2) and 12 months' postpartum (VIHCS wave 3 ) for every child born during screening. Here, we used data from male VAHCS participants who participated in VIHCS. Participants' parents or guardians provided informed written consent at VAHCS recruitment, and participants provided informed verbal consent at subsequent waves. Data collection protocols were approved by the human research ethics committee at the Royal Children's Hospital, Melbourne, Australia.

\section{Measures}

Exposure

Preconception common mental disorder was measured at VAHCS waves 1-7 using the Revised Clinical Interview Schedule (CIS-R), with caseness defined as score $\geq 12$ to identify mixed depression-anxiety symptoms at a level lower than major depressive or anxiety disorder, but which a general practitioner would view as clinically significant. ${ }^{9}$ At VAHCS waves $8-9$, we used the 12-item General Health Questionnaire (GHQ-12) to identify symptoms of psychological distress. ${ }^{10}$ GHQ-12 caseness was defined as standard score $\geq 3,{ }^{11}$ previously found to indicate a CIS-R threshold of $\geq 12 .{ }^{9}$ Continuity of mental disorder was defined as none, adolescent-only, young adult-only, or adolescent and young adult disorder.

\section{Covariates}

Analyses were adjusted for health risk behaviours ( $\geq 20$ drinks on one day in the past week, daily smoking, and weekly cannabis use at one or more adolescent waves), Australian-born/not Australianborn, and participants' parents' education and separation/divorce status.

\section{Outcome}

Antenatal mental health was assessed at VIHCS wave 1, in trimester three of each subsequent pregnancy, using the GHQ-12. ${ }^{9-11}$ 
Table 1 Estimated associations between preconception continuity of common mental disorder and mental health problems in the third trimester of 295 pregnancies to 214 men

\begin{tabular}{|c|c|c|c|c|c|c|c|c|c|c|}
\hline \multirow[t]{3}{*}{ Preconception common mental disorder } & \multicolumn{10}{|c|}{ Antenatal mental health problems } \\
\hline & \multicolumn{4}{|c|}{ Crude risk } & \multicolumn{3}{|c|}{ Unadjusted model } & \multicolumn{3}{|c|}{${ }^{\mathrm{a} A d j u s t e d ~ m o d e l ~}$} \\
\hline & $N$ & $n$ & $\%$ & $(95 \% \mathrm{Cl})$ & $\mathrm{RR}$ & $(95 \% \mathrm{Cl})$ & $P$ & $\mathrm{RR}$ & $(95 \% \mathrm{Cl})$ & $P$ \\
\hline None & 183 & 10 & 5.40 & $(2.00-8.80)$ & ref & & & ref & & \\
\hline Adolescent only & 46 & 4 & 9.70 & $(0.44-18.97)$ & 1.63 & $(0.49-5.41)$ & 0.423 & 1.47 & $(0.45-4.87)$ & 0.525 \\
\hline Young adult only & 34 & 8 & 22.78 & (7.52-38.04) & 3.86 & $(1.37-10.90)$ & 0.011 & 3.75 & $(1.37-10.22)$ & 0.010 \\
\hline Adolescent and young adult & 32 & 9 & 27.90 & $(11.17-44.64)$ & 5.30 & (2.13-13.18) & $<0.001$ & 4.60 & (1.71-12.35) & 0.003 \\
\hline
\end{tabular}

\section{Analysis}

We estimated the prevalence and relative risks of paternal antenatal mental health problems stratified by continuity of preconception common mental disorder, using generalised estimating equations owing to family clustering. We included participants assessed in all phases: adolescence, young adulthood, and antenatal. Missing preconception data were addressed using multiple imputation. Most variables had fewer than $10 \%$ values missing; only four had 10-20\% (common mental disorder, risky drinking, cannabis use and smoking at wave 6). Estimates were obtained by pooling results across 50 imputed data-sets using Rubin's rules. ${ }^{12}$ We used Stata $14.2 .^{13}$

\section{Results}

Supplementary Appendix A, available at https://doi.org/10/1192/ bjo.2017.10, shows the flow of men through VAHCS and VIHCS. Of 332 male VAHCS participants who reported 545 pregnancies during VIHCS recruitment, 214 (65\%) men participated in VIHCS with 295 (55\%) pregnancies. VIHCS participants were less likely to use cannabis weekly in adolescence $(P=0.007)$ or be born outside Australia $(P<0.001)$, and more likely to have at least one parent who completed high school $(P=0.001)$, than those who did not participate, but did not differ on other preconception analysis variables (supplementary Appendix B).

Antenatal mental health problems were reported in 23 (10.7\%, 95\% CI 6.6-14.9) men and 31 (10.5\%, 95\% CI 7.0-14.0) pregnancies. In total, $85(39.7 \%)$ men reported preconception common mental disorder at least once: $35(16.4 \%)$ adolescent only; 25 (11.6\%) young adult only; and $25(11.6 \%)$ both adolescent and young adult (supplementary Appendix C).

In over two-thirds of pregnancies where men reported antenatal mental health problems, there was a history of preconception common mental disorder, compared with around one-third of those without antenatal problems (68.1\% (95\% CI 49.8-86.3) v. $34.3 \%$ (95\% CI $28.3-40.1)$ ). Those with preconception common mental disorder in both adolescence and young adulthood had the greatest risk of antenatal mental health problems (adjusted RR (aRR) 4.60, 95\% CI 1.71-12.35), and those with common mental disorder in young adulthood only were also at increased risk (aRR 3.75, 95\% CI 1.37-10.22) (Table 1).

Men with a history of persistent common mental disorder from adolescence to young adulthood did not go on to experience mental health problems in $23(72.1 \%, 95 \%$ CI $55.4-88.9)$ pregnancies. For ten $(31.9 \%, 95 \%$ CI $14.4-49.4)$ of the 31 pregnancies during which men reported antenatal mental health problems, there was no history of preconception common mental disorder.

\section{Discussion}

Overall, one in ten men reported antenatal mental health problems, similar to a recent meta-analytic estimate of third-trimester prevalence of $9 \%{ }^{1}$ In over two-thirds of these pregnancies, there was a history of common mental disorder prior to conception. Our findings suggest that mental health problems in the transition to parenthood are often a continuation or recurrence of persistent or recent preconception problems.

Nearly three-quarters of men with preconception common mental disorders did not experience antenatal mental health problems. This may reflect the natural resolution of mental health problems for some men during young adulthood. ${ }^{7}$ Previous research has found that, overall, men's mental health does not worsen from preconception to expectant fatherhood. ${ }^{14,15}$ Our findings are consistent in that overall prevalence was similar across the preconception and antenatal windows (supplementary Appendix C), but the lack of prior history in one-third of antenatal cases suggests that risks are heightened for some. Other factors may increase or mitigate risk, including physical health and perceived stress, ${ }^{16}$ partner's mental health, ${ }^{1}$ partner conflict, and social support. ${ }^{17}$

Strengths of this study include the prospective two-generational design, and repeated assessment of common mental disorder across adolescence and young adulthood. Our sample size was small, and results may not be generalisable to trimesters one and two, or to younger and older fathers. Attrition and non-response may have affected results. We used multiple imputation to address biases due to missing VAHCS data. This would not have addressed differential recruitment to VIHCS; nonetheless, prevalence of adolescent common mental disorder did not differ between VIHCS participants and non-participants. We did not use diagnostic measures and our estimates of association are likely to be conservative, as greater continuity might be expected for more severe disorders.

A number of countries have moved to implement or improve maternal mental health screening antenatally, but fathers have yet to be systematically included. ${ }^{18}$ Given that risk for antenatal mental health problems is high for men with a persistent or proximal preconception history, an opportunity exists during pregnancy and the perinatal period to identify and support fathers with preexisting vulnerability. Particularly in light of men's disinclination to proactively seek help for mental health problems, ${ }^{19}$ early intervention to improve adolescent mental health may also yield benefits for their own continuing mental health and that of future generations. $^{6}$ 
Elizabeth Spry, BA (Hons), Research Officer, Centre for Adolescent Health, Murdoch Children's Research Institute, Melbourne, and PhD candidate, Centre for Social and Early Emotional Development, School of Psychology, Faculty of Health, Deakin University, Geelong; Rebecca Giallo, PhD, Senior Research Fellow, Healthy Mothers Healthy Families group, Murdoch Children's Research Institute, Melbourne; Margarita MorenoBetancur, PhD, Postdoctoral research fellow, Clinical Epidemiology and Biostatistics Unit, Murdoch Children's Research Institute, Melbourne, and Melbourne School of . Lecturer in Psychology, School of Psychology, Faculty of Health, Deakin University, and Honorary Research Fellow, University of Melbourne and Murdoch Children's Research Institute; Denise Becker, MBiostats, Biostatistician, Centre for Adolescent Health, Murdoch Children's Research Institute, Melbourne; Rohan Borschmann, PhD, Senior Research Fellow, Centre for Adolescent Health, Murdoch Children's Research Institute, Melbourne, and Honorary Research Fellow, Melbourne School of Population and Global Health, University of Melbourne and Institute of Psychiatry, Psychology \& Neuroscience King's College London, London; Stephanie Brown, PhD, Senior Principal Research Fellow and Head of the Healthy Mothers Healthy Families group, Murdoch Children's Research Institute, Melbourne, and Honorary Professorial Fellow, University of Melbourne; George C. Patton, MD, Professorial Fellow in Adolescent Health Research, University of Melbourne, and Senior Principal Research Fellow, National Health and Medical Research Council; Craig A. Olsson, PhD, Professor and Head of the Centre for Social and Early Emotional Development, School of Psychology, Faculty of Health, Deakin University, and Honorary Principal Research Fellow, University of Melbourne and Murdoch Children's Research Institute, Australia

Correspondence: Elizabeth Spry, Murdoch Children's Research Institute, Royal Children's Hospital, Flemington Road, Parkville 3052, Melbourne, Australia. Email: elizabeth.spry@mcri.edu.au

First received 21 Jul 2017, final revision 7 Nov 2017, accepted 7 Dec 2017

\section{Funding}

This work was supported by the National Health and Medical Research Council; Australian Rotary Health; Colonial Foundation; Perpetual Trustees; Financial Markets Foundation for Children (Australia); Royal Children's Hospital Foundation; Murdoch Children's Research Institute; Australian Postgraduate Award to E.S.; and the Australian Research Council. Research at the Murdoch Children's Research Institute is supported by the Victorian Government's Operational Infrastructure Program.

\section{Acknowledgements}

We thank the families who have participated in the Victorian Adolescent Health Cohort Study and the Victorian Intergenerational Health Cohort Study, and the study research team involved in the collection and management of Victorian Intergenerational Health cohort Study data

\section{Supplementary material}

Supplementary material is available online at https://doi.org/10.1192/bjo.2017.10.

\section{References}

1 Cameron EE, Sedov ID, Tomfohr-Madsen LM. Prevalence of paternal depression in pregnancy and the postpartum: an updated meta-analysis. J Affect Disord 2016; 206: 189-203.
2 Paulson JF, Bazemore SD, Goodman JH, Leiferman JA. The course and interrelationship of maternal and paternal perinatal depression. Arch Womens Ment Health 2016; 19(4): 655-63.

3 Ramchandani PG, Stein A, O'Connor TG, Heron J, Murray L, Evans J. Depression in men in the postnatal period and later child psychopathology: a population cohort study. J Am Acad Child Adolesc Psychiatry 2008; 47(4): 390-8.

4 Gutierrez-Galve L, Stein A, Hanington L, Heron J, Ramchandani P. Paternal depression in the postnatal period and child development: mediators and moderators. Pediatrics 2015; 135(2): e339-47.

5 Frey KA, Navarro SM, Kotelchuck M, Lu MC. The clinical content of preconception care: preconception care for men. Am J Obstet Gynecol 2008; 199(6): S389-95

6 Garfield CF. Supporting fatherhood before and after it happens. Pediatrics 2015; 135(2): e528-30

7 Patton GC, Coffey C, Romaniuk H, Mackinnon A, Carlin JB, Degenhardt L, et al. The prognosis of common mental disorders in adolescents: a 14-year prospective cohort study. Lancet 2014; 383(9926): 1404-11.

8 Australian Bureau of Statistics. 3301.0 - Births, Australia, 2012 (Latest issue 24/10/2013). ABS, 2013.

9 Lewis G, Pelosi AJ, Araya R, Dunn G. Measuring psychiatric disorder in the community: a standardized assessment for use by lay interviewers. Psychol Med 1992; 22(2): 465-86.

10 Goldberg DP, Williams PA. A User's Guide to the General Health Questionnaire. NFER-Nelson, 1988

11 Goldberg DP, Gater R, Sartorius N, Ustun TB, Piccinelli M, Gureje O, et al. The validity of two versions of the $\mathrm{GHQ}$ in the WHO study of mental illness in general health care. Psychol Med 1997; 27(01): 191-7.

12 Rubin DB. Multiple Imputation for Nonresponse in Surveys. John Wiley \& Sons, Inc., 1987.

13 StataCorp. Stata Statistical Software: Release 14. StataCorp LP, 2015

14 Leach LS, Mackinnon A, Poyser C, Fairweather-Schmidt AK. Depression and anxiety in expectant and new fathers: Iongitudinal findings in Australian men. Br J Psychiatry 2015; 206(6): 471-8.

15 Leach LS, Olesen SC, Butterworth P, Poyser C. New fatherhood and psychoIogical distress: a longitudinal study of Australian men. Am J Epidemiol 2014; 180(6): 582-9.

16 Underwood L, Waldie KE, Peterson E, D'Souza S, Verbiest M, McDaid F, et al Paternal depression symptoms during pregnancy and after childbirth among participants in the Growing Up in New Zealand study. JAMA Psychiatry 2017; 74(4): 1-10.

17 Wee KY, Skouteris H, Pier C, Richardson B, Milgrom J. Correlates of ante- and postnatal depression in fathers: a systematic review. J Affect Disord 2011; 130(3): 358-77.

18 Rominov H, Pilkington PD, Giallo R, Whelan TA. A systematic review of interventions targeting paternal mental health in the perinatal period. Infant Ment Health J 2016; 37(3): 289-301.

19 Isacco A, Hofscher R, Molloy S. An examination of fathers' mental health help seeking: a brief report. Am J Mens Health 2016; 10(6): NP33-8. 